\title{
Tacrolimus dye drop treatment for the management of early post-operative intraocular inflammation after therapeutic keratoplasty for severe infectious keratitis
}

\author{
YUNXIAO ZANG ${ }^{1}$, SHANG LI $^{2}$, FANG RUAN ${ }^{2}$, ZHAOCHUAN LIU ${ }^{1}$ and YING JIE ${ }^{1}$ \\ ${ }^{1}$ Beijing Tongren Eye Center, Beijing Tongren Hospital, Beijing Institute of Ophthalmology, \\ Capital Medical University, Beijing 100730; ${ }^{2}$ Department of Ophthalmology, \\ Beijing You'an Hospital, Capital Medical University, Beijing 100069, P.R. China
}

Received September 23, 2019; Accepted June 24, 2020

DOI: $10.3892 / e t m .2020 .9057$

\begin{abstract}
The current study investigated the anti-inflammatory effect of $0.1 \%$ tacrolimus eye drops for the treatment of therapeutic penetrating keratoplasty (TPK) for severe infectious keratitis during early disease stages and reported the long-term clinical outcomes. The present retrospective study included 20 eyes from patients diagnosed with severe keratitis who underwent TPK surgery. Patients were followed-up for up to 12-18 months. Tacrolimus eye drops were administered 4 times/day starting on the first day post-surgery. Glucocorticoid eye drops were subsequently added to treatment plans one-month post-surgery. All patients were followed-up for the first 3 post-operative days, then examined once a week thereafter for the first month. In early post-operative stages, the states of the grafts $(\Delta S)$ and the absorption of intraocular inflammation (S) were observed. $\Delta \mathrm{S}$ was defined as the difference between the states of the grafts on the first post-operative day (SS1d) and those at one month post-surgery (SS1m). S was calculated as the difference between the inflammation score mean one day post-surgery (T1d) and the mean one-month post-surgery (T1m). For long term clinical outcomes, graft failure rates and complications were recorded. Among the 20 eyes analyzed, the mean T1d, T1m and S were 7.4 \pm 2.06 , $2.0 \pm 2.47$ and $5.4 \pm 2.13(\mathrm{P}<0.01)$, respectively. The mean SS1d, $\mathrm{SS} 1 \mathrm{~m}$ and $\Delta \mathrm{S}$ were $5.3 \pm 1.56,3.8 \pm 1.24$ and $1.5 \pm 1.5(\mathrm{P}<0.01)$, respectively. During follow-up, there were 6 cases of corneal graft failure, 4 of which were due to immune rejection and 2 of which were due to complications. The current study concluded that tacrolimus eye drops facilitated the absorption
\end{abstract}

Correspondence to: Dr Ying Jie, Beijing Tongren Eye Center, Beijing Tongren Hospital, Beijing Institute of Ophthalmology, Capital Medical University, 1 Dong Jiao Min Xiang, Dong Cheng, Beijing 100730, P.R. China

E-mail: dr_jieying@163.com

Key words: severe infectious keratitis, therapeutic penetrating keratoplasty, tacrolimus, inflammation, immunosuppressant of intraocular inflammation in the early post-operative period of TPK and may extend long term survival of grafts in cases of severe infectious keratitis.

\section{Introduction}

Patients with severe recalcitrant infectious keratitis exhibit deep ulcers, corneal neovascularization, anterior chamber empyema and a wide range of infections when compared with common infectious corneal ulcers (1). Affected eyes are at risk of severe complications including corneal perforation and spreading of infection to adjacent tissues, which may require subsequent enucleation (2). In cases where medical therapy is insufficient, therapeutic penetrating keratoplasty (TPK) may be promptly considered $(3,4)$. However, due to the poor pre-operative corneal condition, the early post-operative intraocular inflammatory response is more serious than that of non-severe patients (5). Therefore, further research into early post-operative anti-inflammatories is required.

In the majority of TPK cases, glucocorticoid eye drops are the gold standard for early anti-inflammatory post-surgical treatment to reduce intraocular inflammation and prevent immunological graft rejection (6). However, glucocorticoid eye drops may be undesirable and possibly contraindicated due to the increased risk of infection recurrence and lasting infection in patients with severe infectious keratitis following TPK, particularly affecting those with fungal corneal ulcers (7). Therefore, research into alternative anti-inflammatory drugs is required to replace traditional glucocorticoid treatment in patients with severe infectious keratitis that have received TPK.

Tacrolimus, which is a macrolide antibiotic purified from the metabolites of streptavidin, is a novel immunosuppressive agent that exerts immunosuppressive activity by inhibiting calcineurin (8). In ophthalmology, tacrolimus eye drops are mainly administered to prevent rejection after corneal transplantation (9), vernal conjunctivitis (10), refractory inflammatory ocular surface disease (11) and ocular surface problems of graft-versus-host disease (12). Previous research into tacrolimus eye drop treatment following corneal transplantation has aimed to prevent rejection $(9,13,14)$. To the best of our knowledge, no previous study has assessed early intraocular inflammation management. 
The current study retrospectively evaluated the effect of tacrolimus eye drops on post-operative inflammation following TPK treatment in patients with severe infectious keratitis.

\section{Materials and methods}

The current study protocol was approved by the Institutional Review Board of Capital Medical University, Beijing Tongren Hospital and conducted in accordance with the Declaration of Helsinki. Patients provided their written informed consent for enrollment prior to surgery and oral consent was obtained for the analysis and publication of their data. Additionally, patients received an oral explanation regarding the analysis and publication of relevant data.

Inclusion and exclusion criteria. The present retrospective study included 20 patients who underwent TPK surgery between January and May in 2017 in the Beijing Tongren Eye center (Beijing, China). The patients comprised 14 males and 6 females. The average age was $57.1 \pm 10.77$ years (range, 40-70 years). Patients included in the current study underwent TPK and had positive corneal scraping culture results. Patients suspected of bacterial keratitis routinely underwent aerobic culture of the specimen. Corneal scrapings were inoculated onto nutrient broth, blood agar plate $(0.05 \%$ Defibrinated Sheep Blood), MacConkey and Chocolate Agar Plate (0.05\% Defibrinated Sheep Blood) medium (Beijing Solarbio Science \& Technology Co., Ltd.). Subsequently, samples were incubated for 1-2 days at $28^{\circ} \mathrm{C}$ and $50 \%$ humidity and isolated individual colonies. The Kirby-Bauer method (Disc diffusion method) was used to determine antibiotic sensitivity of different antibiotics. The antibiotics tested were all purchased from Beijing Solarbio Science \& Technology Co., Ltd. and included Amikacin $(30 \mu \mathrm{g})$, Ceftazidime (30 $\mu \mathrm{g})$, Cefoxitin $(30 \mu \mathrm{g})$, Ciprofloxacin $(5 \mu \mathrm{g})$, Gentamicin $(120 \mu \mathrm{g})$, Ofloxacin $(5 \mu \mathrm{g})$, Levofloxacin $(5 \mu \mathrm{g})$, Rifampicin $(5 \mu \mathrm{g})$, Moxifloxacin $(5 \mu \mathrm{g})$, Gatifloxacin $(5 \mu \mathrm{g})$, Oxacillin $(1 \mu \mathrm{g})$. Potato Dextrose Agar (PDA) (Beijing Luqiao Technology Co., Ltd.) was selected as medium for specimens suspected as fungal keratits. Specimens were placed in Thermostatic-Humidistat Cultivating Box at $28^{\circ} \mathrm{C}$ and $40 \%$ humidity for 3 to 10 days. After that, the colonies in the positive specimens were isolated and transferred to PDA dishes (Tianjin Jinzhang Company). The Rosco paper diffusion method (Neo-Sensitab drug sensitive paper; Rosco Diagnostica) was used for drug sensitivity test. Test drugs include: Natamycin (50 $\mu \mathrm{g}$; Rosco Diagnostica), Terbinafine (30 $\mu \mathrm{g}$; Rosco Diagnostica), Itraconazole (8 $\mu \mathrm{g}$; Rosco Diagnostica), Fluconazole (15 $\mu \mathrm{g}$; Rosco Diagnostica), Amphotericin B (15 $\mu \mathrm{g}$, Rosco Diagnostica), Voriconazole ( $1 \mu \mathrm{g}$; Rosco Diagnostica). The diagnostic criteria of severe infectious keratitis (1) was as follows: Corneal ulcer area $>6 \mathrm{~mm}^{2}$ or lesion depth $>2 / 3$ of corneal thickness; presence of anterior chamber exudate or empyema; and corneal ulcer perforation or descemetocele. The criteria for surgery for patients with severe infectious keratitis (15) were: No healing after 7 days of standard treatment; descematocele with infiltrates; and corneal perforation $>3 \mathrm{~mm}$ with active inflammation. All corneal transplants were performed by the same surgeon (JY) in Beijing Tongren Eye center (Beijing, China). Excluded patients had a history of previous corneal transplant and/or had congenital eye pathologies, such as cornea dystrophy or leucoma.

Patient information. The patients were diagnosed with bacterial keratitis $(n=8)$ or fungal keratitis $(n=12)$. Each diagnosis was supported by a positive culture in all cases. Pre-operative assessment indicated that the patients exhibited iris adhesions $(n=4)$, high intraocular pressure $(n=7)$ and anterior chamber empyema $(\mathrm{n}=12)$. Additionally, 17 eyes presented with pre-operative corneal neovascularization and a single eye exhibited descemetocele. The mean recipient bed and recipient graft were $7.8 \pm 1.3$ and $8.3 \pm 1.2$, respectively. Pre-operative data are presented in Table I. Due to corneal ulcers problems, Digital Tonometry was used as intraocular pressure measurement. (Tn represented normal pressure. $\mathrm{T}+1, \mathrm{~T}+2$ and $\mathrm{T}+3$ indicated different degrees of intraocular pressure increase, with $\mathrm{T}+3$ as the highest). Corneal perforation or descemetocele was denoted as ' 1 point', and the absence of these was denoted as ' 0 points'. A lack of iris adhesion was recorded as ' 0 point'. If iris adhesion was it was assigned a score of 1 to 4 quadrants, which was correspondingly recorded as '1-4 points'. The height of hypopyon (h) was based on the vertical diameter of the cornea. Ulcer depth was based on central cornea thickness. Ulcer size, ulcer depth and the height of hypopyon were measured using Image Processing Software (vision 11.0; EdgeView, Apple Corporation). TPK sites were divided into orthotopic $(\mathrm{O})$ and partial $(\mathrm{P})$ transplantation. Corneal vascularization was divided into $1-4: 1, \leq 25 \%$ corneal diameter; $2, \leq 50 \%$ corneal diameter; $3, \leq 75 \%$ corneal diameter; $4, \leq 100 \%$ corneal diameter. For example, $(2,1)$ represents corneal vascularization involving 2 quadrants, $<25 \%$ of the corneal diameter.

Anterior chamber washout and penetrating keratoplasty. All corneal transplants were performed with similar technique by the same surgeon at Tongren Eye Hospital $(14,16)$. Donor corneas were stored in OptiSol storage media (Bausch \& Lomb Co., Ltd.) for 7-10 days at $4^{\circ} \mathrm{C}$. The host trephine size was selected to completely cover the infiltrate edge of the ulcer and was oversized by $0.25 \mathrm{~mm}$. Anterior chamber washout was performed in patients with anterior chamber hypopyon as previously described by Jia et al (17). Furthermore, patients with synechia or severe anterior chamber empyema prior to surgery underwent peripheral iridotomy at the 12 o'clock position at the time of surgery (18). The cornea grafts were sutured to the host with 16 interrupted 10-0 monofilament nylon sutures. Suture knots were trimmed and buried toward the donor side. In all cases, the excised host cornea was sent for microbiologic and histopathologic examination in Beijing Tongren Eye center (Beijing, China).

Post-operative medication regimen. Selection of the appropriate antibiotic eye drops and topical antimicrobial agents was based on the microbiological profile and specific sensitivities of the causative infective agent (19).

Quinolone eye drops were administered to all patients as the culture results indicated that gram positive cocci infections were present. The current study administered $5 \mathrm{ml} 0.5 \%$ gatifloxacin eye drops (Anhui Shuangke Pharmaceutical Co., Ltd.) 


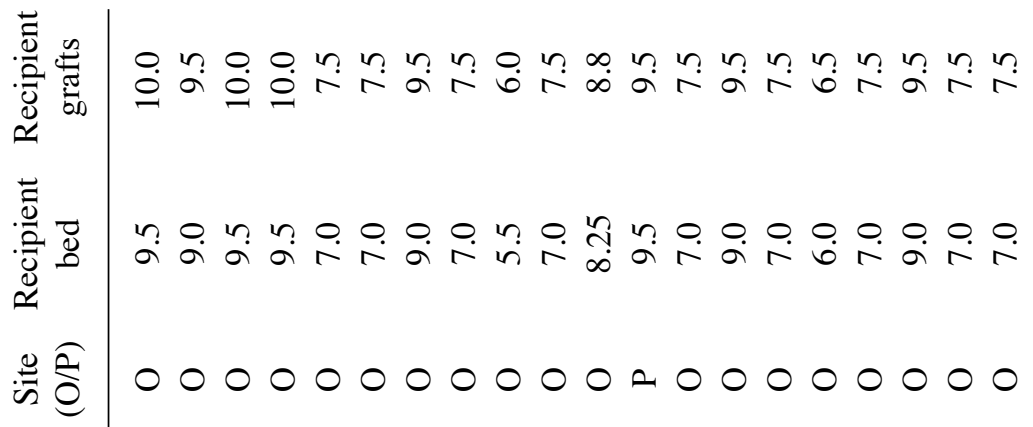

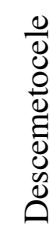

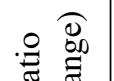

$000000000-0000000000$

焉离

$\vec{i}=0$ 至

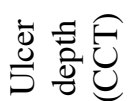

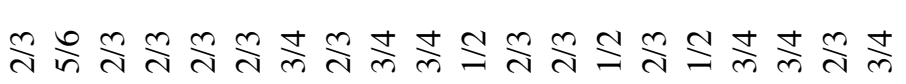

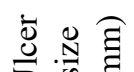

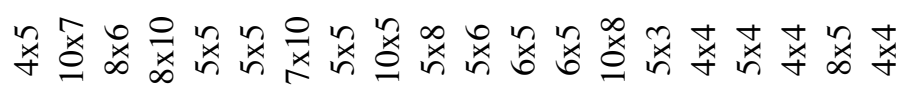

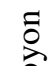

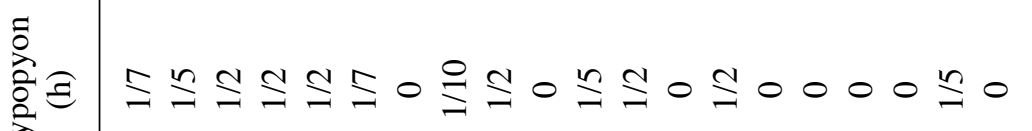
空

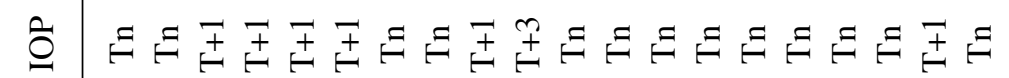
旁 $00-00-000+00000000-0$

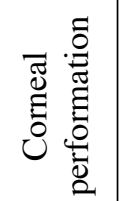
00000000000000000000

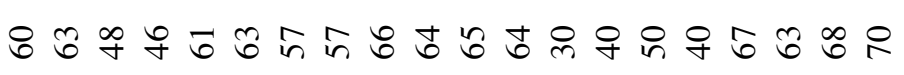


Table II. Intraocular inflammation and graft survival state evaluation criteria.

\begin{tabular}{|c|c|c|c|c|c|c|c|}
\hline Score & Graft clarity & Graft edema & Neovascularization & $\begin{array}{c}\text { Anterior } \\
\text { chamber flare }\end{array}$ & $\begin{array}{c}\text { Anterior } \\
\text { chamber } \\
\text { cells, } \mathrm{n}\end{array}$ & Hyphema & Exudation \\
\hline 0 & Clear cornea, & None & None & None & $<5$ & 0 & N/A \\
\hline 1 & $\begin{array}{l}\text { Slight haze, } \\
\text { details of iris } \\
\text { clearly visible }\end{array}$ & $\begin{array}{l}\text { Mild stromal } \\
\text { thickness }\end{array}$ & $\begin{array}{l}\text { Neovascularization of } \\
\text { peripheral cornea }\end{array}$ & Faint & $6-15$ & $0-1 / 3$ & $\begin{array}{l}\text { Yarn-like } \\
\text { exudation }\end{array}$ \\
\hline 2 & $\begin{array}{l}\text { Increased haze, } \\
\text { some details of } \\
\text { iris no longer } \\
\text { visible }\end{array}$ & $\begin{array}{l}\text { Diffuse stromal } \\
\text { edema }\end{array}$ & $\begin{array}{l}\text { Neovascularization } \\
\text { appearing in the } \\
\text { graft periphery }\end{array}$ & $\begin{array}{l}\text { Moderate (iris } \\
\text { and lens } \\
\text { details clear) }\end{array}$ & $16-25$ & $1 / 3-2 / 3$ & $\begin{array}{l}\text { Cluster-like } \\
\text { exudation }\end{array}$ \\
\hline 3 & $\begin{array}{l}\text { Advanced haze, } \\
\text { pupil still } \\
\text { recognizable }\end{array}$ & $\begin{array}{l}\text { Pronounced edema } \\
\text { with small bleb of } \\
\text { epithelium }\end{array}$ & $\begin{array}{l}\text { Neovascularization } \\
\text { extending deeper }\end{array}$ & $\begin{array}{l}\text { Marked (iris } \\
\text { and lens } \\
\text { details hazy) }\end{array}$ & $26-50$ & $2 / 3-1$ & $\begin{array}{l}\text { Transparent mash } \\
\text { in front of the } \\
\text { lens or behind the } \\
\text { cornea }\end{array}$ \\
\hline 4 & $\begin{array}{l}\text { Opaque cornea } \\
\text { without view of } \\
\text { anterior chamber }\end{array}$ & $\begin{array}{l}\text { Bullous } \\
\text { keratopathy }\end{array}$ & $\begin{array}{l}\text { Neovascularization } \\
\text { extending to the } \\
\text { entire graft }\end{array}$ & $\begin{array}{l}\text { Intense (fibrin or } \\
\text { plastic aqueous) }\end{array}$ & $>50$ & N/A & $\begin{array}{l}\text { Pre-iridal } \\
\text { adhesion or } \\
\text { Post-iridal } \\
\text { adhesion }\end{array}$ \\
\hline
\end{tabular}

N/A, not applicable.

4 times/day with 5 g $0.3 \%$ gatifloxacin gel (Shenyang Xingqi Eye Medicine Co., Ltd.) 3 times/day for 2 weeks then gradually reduced the amount according to corneal condition.

All cases of fungal infection were treated with $5 \mathrm{ml} \mathrm{5 \%}$ natamycin eye drops (Hubei Pharmaceutical Co., Ltd.) and $0.5 \%$ fluconazole eye drops (Yongguang Pharmaceutical Co., Ltd.) 4 times/day for 2 weeks then gradually reduced the amount according to corneal condition.

Furthermore, $5 \mathrm{mg} 0.1 \%$ tacrolimus eye drops (Senju Pharmaceutical Co., Ltd.) were applied 4 times/day for 2 months then gradually reduced the amount according to corneal condition starting on the first day post-surgery for all patients.

Steroid eye drops were not administered during the first post-operative month. However, after a period of one month, if no signs of active inflammation were present, $1 \%$ prednisolone acetate (Allergan Pharmaceutical Co., Ltd.) was added to the treatment regimen and applied 4 times/day for a month then gradually reduced the amount according to corneal condition. Additionally, $0.3 \%$ tobramycin $/ 0.1 \%$ dexamethasone eye ointment (S.A. Alcon Couvreur Pharmaceutical Co., Ltd.) was applied one time at night for one month (20). After 6 months, the frequency of prednisolone eye drops was reduced to twice/day and after one year, to once/day. Prednisolone was stopped after a period of two years.

Follow-up. Eyes were examined daily using a slit lamp (YZ5S digital slit lamp microscope, Shanghai Hanfei Medical Equipment Co., Ltd.) for the first 3 post-operative days, then re-examined once a week and at weekly intervals thereafter for the first month. Routine examination included uncorrected visual acuity (Snellen visual acuity chart), intraocular pressure and slit biomicroscopy examinations. Icare rebound tonometer (TAO1i; Icare Finland Oy) was selected for intraocular pressure measurement. Digital tonometry was selected if Icare couldn't get results due to cornea condition. Slit biomicroscopy was operated by the same skilled ophthalmologist.

Observation of early post-operative inflammatory response. The absorption of intraocular inflammation in the early post-operative period (one month) was assessed under a slit lamp biomicroscopy. All examinations were operated by the same ophthalmologist who performed the surgeries. The following parameters were assessed. The scoring of aqueous flare, aqueous cells, cellulose exudation and hyphema presence were graded according to the uveitis inflammation scoring criteria (21). The graft transparency, degree of graft edema and corneal neovascularization were graded according to the cornea rejection scoring criteria (22). These items included graft clarity, graft edema, neovascularization, anterior chamber flare, anterior chamber cells and exudation received between 0-4 points. Hyphema received a score of between 0-3 points. When the signs were more severe, the scores were higher. The grading systems are summarized in Table II.

$\mathrm{S}$ was defined as the post-operative early intraocular inflammation absorption fraction and was calculated as the difference between the post-exudation scores on the first day (T1d) and after one month (T1m; S=T1d-T1m). Additionally, $\Delta S$ was defined as the graft survival state and is the difference between the graft state on the first post-operative day (SS1d) and one month post-surgery (SS1m).

Long-term PK results. All 20 patients were followed up for 12-18 months. Visual acuity, intraocular pressure and thorough 
Table III. Post-operative intraocular inflammatory response and graft survival state scores.

\begin{tabular}{|c|c|c|c|c|c|c|}
\hline Patient no. & T1d & $\mathrm{T} 1 \mathrm{~m}$ & S & SS1d & $\mathrm{SS} 1 \mathrm{~m}$ & $\Delta \mathrm{S}$ \\
\hline 1 & 10 & 2 & 8 & 6 & 4 & 2 \\
\hline 2 & 7 & 0 & 7 & 5 & 3 & 2 \\
\hline 3 & 7 & 4 & 3 & 6 & 3 & 3 \\
\hline 4 & 10 & 4 & 6 & 7 & 4 & 3 \\
\hline 5 & 10 & 6 & 4 & 3 & 2 & 1 \\
\hline 6 & 5 & 0 & 5 & 4 & 2 & 2 \\
\hline 7 & 8 & 0 & 8 & 3 & 2 & 1 \\
\hline 8 & 5 & 0 & 5 & 6 & 2 & 4 \\
\hline 9 & 8 & 4 & 4 & 7 & 5 & 2 \\
\hline 10 & 5 & 4 & 1 & 7 & 5 & 2 \\
\hline 11 & 4 & 1 & 3 & 2 & 5 & -3 \\
\hline 12 & 5 & 2 & 3 & 4 & 4 & 0 \\
\hline 13 & 7 & 0 & 7 & 7 & 6 & 1 \\
\hline 14 & 9 & 1 & 8 & 5 & 4 & 1 \\
\hline 15 & 6 & 0 & 6 & 5 & 4 & 1 \\
\hline 16 & 9 & 2 & 7 & 7 & 4 & 3 \\
\hline 17 & 6 & 0 & 6 & 5 & 3 & 2 \\
\hline 18 & 7 & 0 & 7 & 4 & 4 & 0 \\
\hline 19 & 11 & 9 & 2 & 6 & 6 & 0 \\
\hline 20 & 9 & 1 & 8 & 7 & 4 & 3 \\
\hline Mean & $7.4 \pm 2.06$ & $2.0 \pm 2.47$ & $5.4 \pm 2.13$ & $5.3 \pm 1.56$ & $3.8 \pm 1.24$ & $1.5 \pm 1.50$ \\
\hline P-value & & & $<0.01$ & & & $<0.01$ \\
\hline
\end{tabular}

T1d, post-operative exudation score on day $1 ; \mathrm{T} 1 \mathrm{~m}$, post-operative exudation score after $1 \mathrm{month}$; S, post-operative early intraocular inflammation absorption fraction; SS1d, graft state on day 1 ; SS1m, graft state after 1 month; $\Delta \mathrm{S}$, graft survival state.

slit lamp eye exams were performed at every visit and symptoms and complications were documented.

Statistical methods. SPSS software (version 23.0; IBM Corp) was used for statistical analysis. Data were tested for normality using the Kolmogorov-Smirnov test and provided as mean and standard deviation (SD). A paired sample t-test was used to compare the difference between T1d and T1m and the difference between SS1d and SS1m. P $<0.05$ was considered to indicate a statistically significant difference.

\section{Results}

Case data analysis. Post-operative inflammatory responses (T1d, T1m and S) and graft survival state scores (SS1d, SS1m and $\Delta S$ ) are summarized in Table III. The average score of intraocular inflammation was $7.4 \pm 2.06$ on the first day and $2.0 \pm 2.47$ by the first post-operative month. The mean of $S$ was $5.4 \pm 2.13$. This difference was statistically significant $(\mathrm{P}<0.01)$. The average of SS1d was $5.3 \pm 1.56$ and $3.8 \pm 1.24$ for SS1m. $\Delta S$ was also statistically significant $(\mathrm{P}<0.01)$ Early postoperative anterior images of 2 patients are depicted in Figs. 1 and 2.

Long-term TPK follow-up. Of the 20 patients, 6 cases resulted in transplant failure. In these cases, 4 were due to rejection, a single case was due to corneal epithelial defects and a further case was caused by uncontrolled infection recurrence. Patient follow-up times and final outcomes are presented in Table IV. The survival rates of the grafts are presented in Fig. 3. At 4 months post-surgery, one patient exhibited transplant rejection leading to graft failure. Two cases exhibited failure at 8 months post-surgery, one of which was due to persistent epithelial defects. There were three cases of graft failure at 11 months post-surgery, two of which were due to rejection and one failed due to the recurrence of fungal infection.

Visual acuity prognosis. The pre-operative visual acuity examination for all 20 patients was light perception. Post-operative visual acuity increased in 16 patients, decreased in 2 patients and 2 patients maintained pre-operative visual acuity. The specific vision changes prior to surgery and the best measured uncorrected visual acuity during the follow-up period are presented in Fig. 4. The pre-operative visual acuity of all patients was $<0.05$ and the optimal visual acuity after surgery was improved compared with that before surgery.

An association between early post-operative inflammatory absorption and long-term rejection based on a 12-month follow-up period of 20 patients was observed and is demonstrated in Fig. 5. In the 4 patients who exhibited transplant rejection, the early inflammatory absorption 


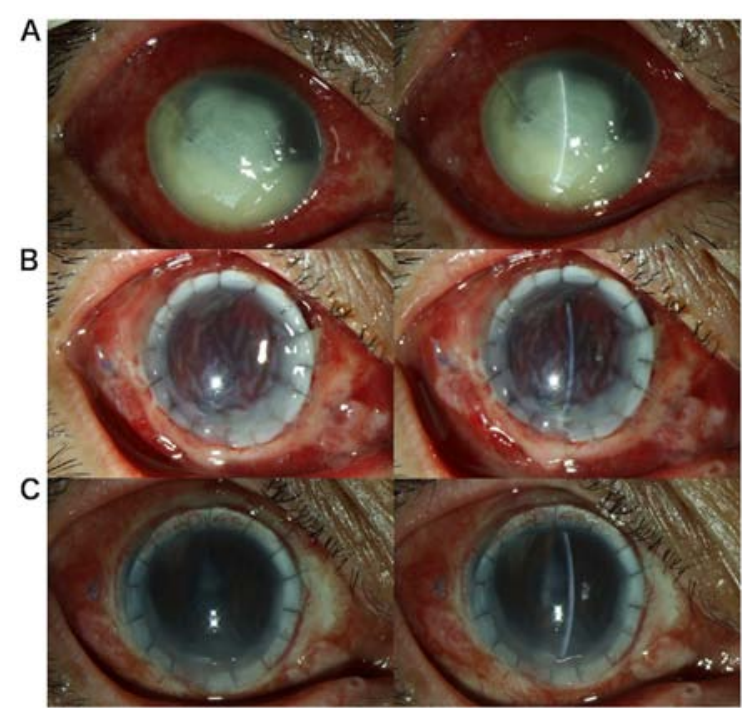

Figure 1. A severe fungal ulcer caused by Fusarium solani. (A) Pre-operation image. The area of the ulcer was $\sim 8 \times 10 \mathrm{~mm}$ and the anterior chamber empyema height was $2 / 3$ corneal thickness. (B) First day post-surgery. Ciliary congestion, corneal edema, unclear iris texture and anterior chamber cellulose exudation were observed. (C) One-month post-surgery. Ciliary congestion is relieved, the cornea is transparent, the iris texture is clear and the anterior chamber is exuded and absorbed.

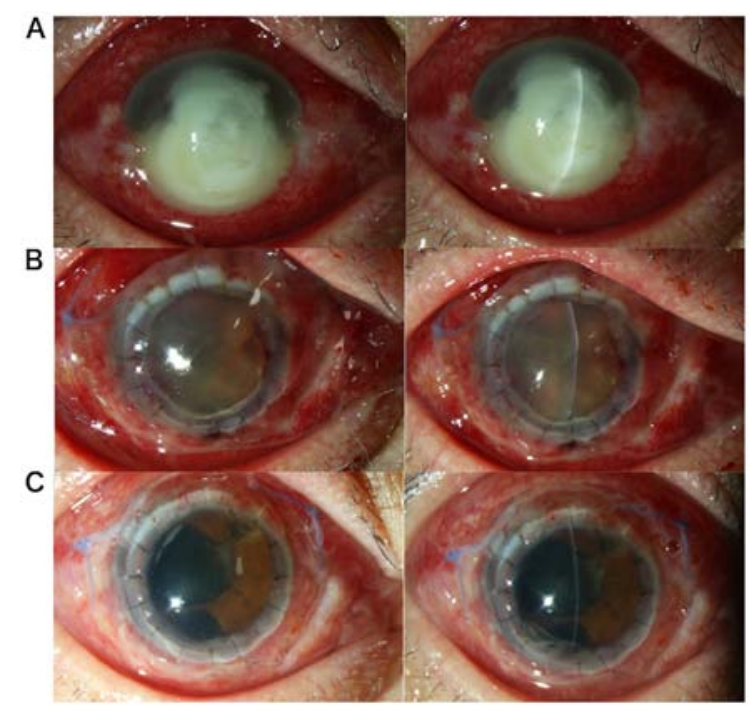

Figure 2. A severe bacterial ulcer caused by Staphylococcus aureus. (A) Pre-operation image. The area of the corneal ulcer is $\sim 8 \times 6 \mathrm{~mm}$ and the anterior chamber empyema height is $1 / 2$ corneal thickness. (B) First day post-surgery. Ciliary congestion, corneal edema, unclear iris texture, rear elastic layer wrinkles and anterior chamber hemorrhage are observed (C) One-month post-surgery. Ciliary congestion is relieved, cornea is transparent and anterior chamber is exuded and absorbed.

scores were relatively low, suggesting that long-term graft rejection may have a relationship with poor early inflammation control.

Complications during post-operative follow-up. Post-operative complications included a persistent corneal epithelial defect that ultimately led to failure. A total of 3 cases were diagnosed with secondary fungal infections (at 6,8 and 11 months after surgery), of which 2 cases were medically controlled and a

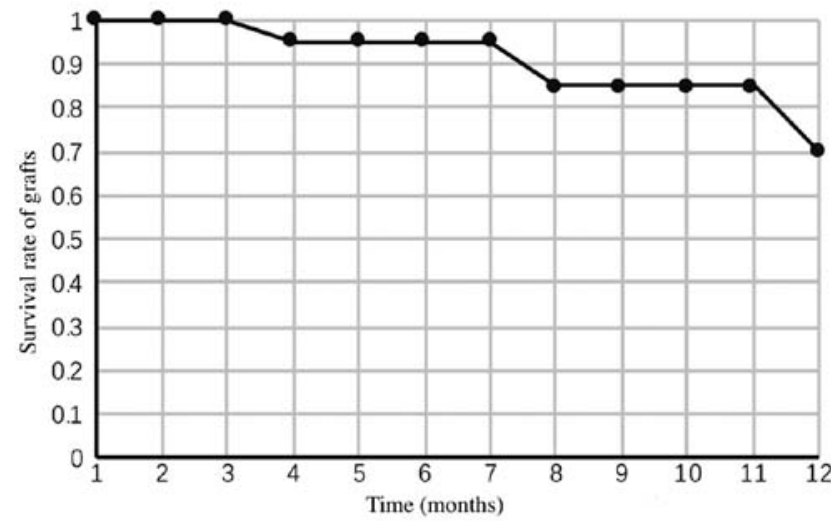

Figure 3. Survival rate of the grafts during follow-up at 12 months post-surgery.

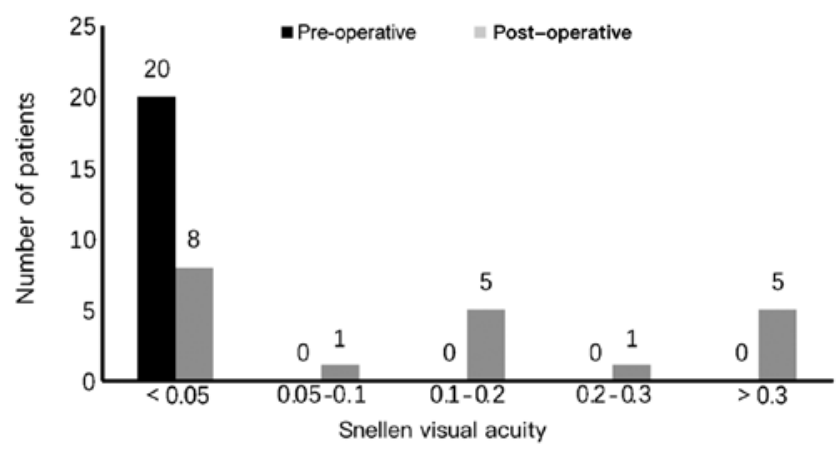

Figure 4. Pre-operative and post-operative visual acuity of 20 patients with severe keratitis. The pre-operative visual acuity of all patients was $<0.05$ and the optimal visual acuity after surgery was improved compared with that before surgery.

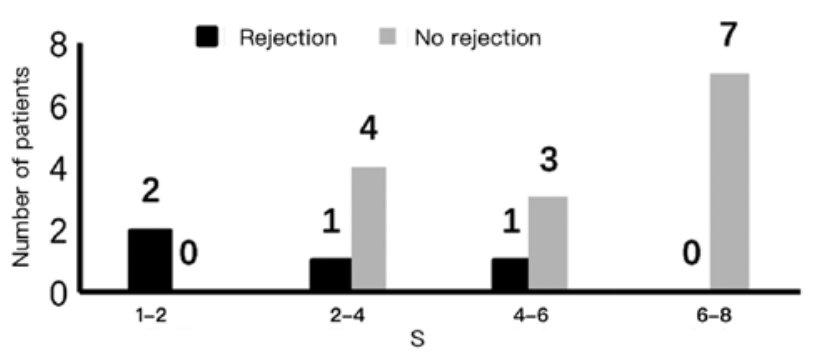

Figure 5. Association between the degree of inflammatory exudation and long-term immune rejection in the early post-operative period. Absorption of inflammation index score in patients with immunological rejection of the graft, the early inflammatory absorption score was between 1-6 points, while the patients with no immune rejection had an absorption score between 2-8.

single case resulted in graft failure. Additionally, one patient developed secondary glaucoma and lost light perception in that eye. One patient with a $9.5 \mathrm{~mm}$ graft had persistent epithelial defects which ultimately healed by wearing a soft bandage contact lens.

\section{Discussion}

The current retrospective study utilized tacrolimus eye drops in patients with severe infectious keratitis post-TPK at Beijing Tongren Hospital. During post-operative follow-up, it was 
Table IV. Patient follow-up times and prognosis.

\begin{tabular}{|c|c|c|}
\hline Case (no.) & Follow-up timepoint (weeks) & Prognosis \\
\hline 1 & $1 / 7,2 / 7,3 / 7,1,2,3,4,12,32,33$ & $\begin{array}{l}\text { Occured at } 32 \text { nd week after surgery; persistent epithelial defects; } \\
\text { resulting in failure of grafting; epithelial being stable by wearing } \\
\text { bandage soft contact lens }\end{array}$ \\
\hline 2 & $1 / 7,2 / 7,3 / 7,1,2,3,4,12,24,36$ & No graft failure or complications and the graft kept clear \\
\hline 3 & $1 / 7,2 / 7,3 / 7,1,2,3,4,12,24,35,70$ & No graft failure or complications and the graft kept clear \\
\hline 4 & $1 / 7,2 / 7,3 / 7,1,2,3,4,48,49$ & $\begin{array}{l}\text { Occurred at } 48 \text { th weeks after surgery; endothelial rejection; reversal } \\
\text { after drug treatment }\end{array}$ \\
\hline 5 & $1 / 7,2 / 7,3 / 7,1,2,3,4,12,24,37,72$ & No graft failure or complications and the graft kept clear \\
\hline 6 & $1 / 7,2 / 7,3 / 7,1,2,3,4,32,33$ & $\begin{array}{l}\text { Occurred at the 16th week after surgery; epithelial rejection; reversal } \\
\text { after drug treatment }\end{array}$ \\
\hline 7 & $1 / 7,2 / 7,3 / 7,1,2,3,4,12,24,48$ & No graft failure or complications and the graft kept clear \\
\hline 8 & $1 / 7,2 / 7,3 / 7,1,3,4,12,24,49$ & No graft failure or complications and the graft kept clear \\
\hline 9 & $1 / 7,2 / 7,3 / 7,1,2,3,4,12,24,48$ & No graft failure or complications and the graft kept clear \\
\hline 10 & $1 / 7,2 / 7,3 / 7,1,2,3,4,12,48,49,72$ & $\begin{array}{l}\text { Occurred at the 48th week after surgery; endothelial rejection; } \\
\text { secondary glaucoma; abandon treatment }\end{array}$ \\
\hline 11 & $1 / 7,2 / 7,3 / 7,1,2,3,4,12,24,48$ & No graft failure or complications and the graft kept clear \\
\hline 12 & $1 / 7,2 / 7,3 / 7,1,2,3,4,12,22,45$ & No graft failure or complications and the graft kept clear \\
\hline 13 & $1 / 7,2 / 7,3 / 7,1,2,3,4,12,24,70$ & no graft failure or complications and the graft kept clear \\
\hline 14 & $1 / 7,2 / 7,3 / 7,1,2,3,4,12,24,47$ & No graft failure or complications and the graft kept clear \\
\hline 15 & $1 / 7,2 / 7,3 / 7,1,2,3,4,6,7$ & $\begin{array}{l}\text { Occurred at } 32 \mathrm{nd} \text { week after surgery; secondary infection; resulting in } \\
\text { failure of grafting; secondary transplantation after drug treatment }\end{array}$ \\
\hline 16 & $1 / 7,2 / 7,3 / 7,1,2,3,4,12,24,48$ & No graft failure or complications and the graft kept clear \\
\hline 17 & $1 / 7,2 / 7,3 / 7,1,2,3,4,12,24,71$ & No graft failure or complications and the graft kept clear \\
\hline 18 & $1 / 7,2 / 7,3 / 7,1,2,3,4,12,24,48$ & No graft failure or complications and the graft kept clear \\
\hline 19 & $1 / 7,2 / 7,3 / 7,1,2,3,4,12,24,48,49,70$ & $\begin{array}{l}\text { Occurred at the 48th week after surgery, endothelial rejection, reversal } \\
\text { after drug treatment }\end{array}$ \\
\hline 20 & $1 / 7,2 / 7,3 / 7,1,2,3,4,12,23,50$ & No graft failure or complications and the graft kept clear \\
\hline
\end{tabular}

determined that the drops had an effect on intraocular inflammation absorption. By reviewing post-operative follow-up patient records (vision and corrected vision, intraocular pressure and anterior angiography), a post-operative intraocular inflammatory management effect of tacrolimus eye drops was observed. According to patient follow-up time, the condition of the corneal grafts was monitored and anti-infectives were ceased after the second week. Observation then continued for 2 weeks until the risk of infection recurrence had been reduced to very low. At this time, due to the risk of rejection in high-risk corneal transplantation, glucocorticoids eye drops were gradually added to the treatment regimen (23). A period of one month post-surgery was a turning point in terms of drug use and changes in the condition and in this turning point inflammation of patients was absorbed significantly. Due to this, one-month post-TPK was defined as an early post-operative time point in the current study.

Severe post-operative inflammation can result from an underlying corneal infection and can increase due to severe neovascularization of the cornea prior to surgery (24) and large corneal grafting (25). To reduce intraocular inflammation, corticosteroids were the preferred anti-inflammatory drug (19). Intraocular injection of tissue plasminogen activator may also promote anterior chamber exudation following TPK (26).
However, for infectious keratitis, especially fungal keratitis, glucocorticoid drugs are contraindicated considering the recurrence of infection after TPK (15).

$\mathrm{Yu}$ et al (27) demonstrated in vitro that tacrolimus significantly reduced pro-inflammatory cytokine expression and increased the anti-inflammatory cytokine expression in lipopolysaccharide-induced keratitis. Sakuma et al (28) also revealed that tacrolimus serves anti-inflammatory and immunosuppressive roles by inhibiting the release of interleukin (IL)-2, IL-3, IL-4, interferon $\gamma$ and granulocyte-macrophage colony stimulating factor and by inhibiting IL-2 receptor expression in T-helper (Th) cells. Tacrolimus treatment has demonstrated significant cytokine production inhibition when compared with steroids (28). Furthermore, when compared with glucocorticoid eye drops, which inhibit multiple pathways of the immune process (29) by modulating the production of certain proteins including lipocortin and enzymes, tacrolimus eye drops primarily inhibit Th1/Th2 cell cytokines by inhibiting calcium- and calmodulin-dependent dephosphatase activity (30). This, in turn, inhibits $\mathrm{T}$ and $\mathrm{B}$ cell activation, as well as other inflammatory cells (30). This mechanism has reportedly resulted in fewer side effects when compared with glucocorticoid eye drops (22). 
The results of the current study demonstrated that tacrolimus eye drops promoted the absorption of early post-operative intraocular inflammation, primarily manifested as the reduction of anterior chamber flare and exudation. Previous study (31) have revealed that the continued aggravation of the aqueous flare may be associated with a high incidence of graft rejection. The present study revealed that patients with long-term rejection post-surgery exhibited less intraocular inflammation absorption scores in the early post-operative period.

Reinfection may present as a milky white, tongue- and endothelium-like growth at the junction of the implant and the graft (32). Shi et al (7) reported a post-operative reinfection rate of $6.34 \%$ in 899 patients with fungal keratitis who underwent TKP or lamellar keratoplasty. Among these patients, PK accounted for $6.79 \%$ of 899 patients and a higher rate of recurrence was identified in those with preoperative hypopyon (10.90\%), corneal perforation $(12.00 \%)$, corneal infection expanding to limbus $(20.69 \%)$ or lens infection with extracapsular cataract extraction $(50 \% ; \mathrm{P}<0.05)$. In the current study, there were 3 cases of fungal infection recurrence post-surgery. All 3 patients exhibited varying degrees of neovascularization prior to the operation of which 2 had anterior chamber hypopyon. There was no increase in recurrence rate. It was speculated that this may be associated with performing two bacterial cultures before and during the operation, and the reasonable use of antibacterial/fungal drugs after TPK.

Dandona et al (33) reported that a higher relative risk of graft failure was associated with host cornea vascularization prior to transplantation in 1,725 cases for corneal transplantation in India. Weisbrod et al (34) revealed that corneal graft failure was associated with pathogeny for corneal transplantation, pre-operative pre-iridal adhesion and the presence of post-operative neovascularization. In a study of 116 cases of fungal keratitis, Li et al (35) demonstrated that when large-diameter TPK ( $\geq 8 \mathrm{~mm}$ ) was performed, there was a high incidence of failure. The primary reasons were graft rejection and secondary glaucoma. In the current study, long-term follow-up post-surgery revealed 6 cases of corneal transplant failure, 4 of which were caused by rejection mediated by immune factors from tacrolimus and corticosteroids eye drop use. Rejection rate has been reported to be associated with the perioperative inflammatory response of the patient and large corneal lesions prior to TPK (36). A previous case study of 134 patients post-TPK for infectious keratitis by Sukhija and Jain (37) reported the most common post-operative complications, which included secondary glaucoma, non-healing epithelial defects and re-infection. Additionally, glaucoma occurred more frequently in patients with pre-operative perforated ulcers and epithelial defects were associated with grafts $>9 \mathrm{~mm}$. In the current study, a single patient with a $9.5 \mathrm{~mm}$ graft had persistent epithelial defects which ultimately healed by wearing a soft bandage contact lens. Additionally, a second patient who had pre-operative perforated ulcers developed glaucoma and eventually lost light perception. In the present study, it was demonstrated that tacrolimus eye drops facilitated the absorption of intraocular inflammation in the early post-operative period of TPK and extend long term survival of grafts in cases of severe infectious keratitis. Future researches of multi-center including large samples were needed.

\section{Acknowledgements}

Not applicable.

\section{Funding}

No funding was received.

\section{Availability of data and materials}

The datasets used and/or analyzed during the current study are available from the corresponding author on reasonable request.

\section{Authors' contributions}

YZ organized data, performed statistical analysis and wrote the manuscript. SL and FR collected images and raw data. ZL collected and interpreted the data and revised manuscript critically for important intellectual content. YJ revised the manuscript, made substantial contributions to conception and design of the study and supplied the patient data used the present study. All authors read and approved the final manuscript.

\section{Ethics approval and consent to participate}

The current study was approved by the Ethics Committee of Beijing Tongren Hospital.

\section{Patient consent for publication}

The patient, guardian or next of kin (in case of deceased patients) provided written informed consent for the publication of any associated data and accompanying images.

\section{Competing interests}

The authors declare that they have no competing interests.

\section{References}

1. Jones DB: Decision-making in the management of microbial keratitis. Ophthalmology 88: 814-820, 1981.

2. Yang JW, Lin HC, Hsiao $\mathrm{CH}$ and Chen PY: Therapeutic penetrating keratoplasty in severe infective keratitis using glycerol-preserved donor corneas. Cornea 31: 1103-1106, 2012.

3. Ti SE, Scott JA, Janardhanan P and Tan DT: Therapeutic keratoplasty for advanced suppurative keratitis. Am J Ophthalmol 143: 755-762, 2007.

4. Sharma N, Sachdev R, Jhanji V, Titiyal JS and Vajpayee RB: Therapeutic keratoplasty for microbial keratitis. Curr Opin Ophthalmol 21: 293-300, 2010.

5. Cohen RA, Gebhardt BM and Bazan NG: A platelet-activating factor antagonist reduces corneal allograft inflammation and neovascularization. Curr Eye Res 13: 139-144, 1994.

6. Rahman I, Carley F, Hillarby C, Brahma A and Tullo AB: Penetrating keratoplasty: Indications, outcomes, and complications. Eye (Lond) 23: 1288-1294, 2009.

7. Shi W, Wang T, Xie L, Li S, Gao H, Liu J and Li H: Risk factors, clinical features, and outcomes of recurrent fungal keratitis after corneal transplantation. Ophthalmology 117: 890-896, 2010.

8. MacMillan D: FK506 binding proteins: Cellular regulators of intracellular Ca2+ signalling. Eur J Pharmacol 700: 181-193, 2013.

9. Magalhaes OA, Marinho DR and Kwitko S: Topical 0.03\% tacrolimus preventing rejection in high-risk corneal transplantation: A cohort study. Br J Ophthalmol 97: 1395-1398, 2013. 
10. Wan Q, Tang J, Han Y, Wang D and Ye H: Therapeutic effect of $0.1 \%$ tacrolimus eye drops in the tarsal form of vernal keratoconjunctivitis. Ophthalmic Res 59: 126-134, 2018.

11. Lee YJ, Kim SW and Seo KY: Application for tacrolimus ointment in treating refractory inflammatory ocular surface diseases. Am J Ophthalmol 155: 804-813, 2013.

12. Tam PM, Young AL, Cheng LL and Lam PT: Topical $0.03 \%$ tacrolimus ointment in the management of ocular surface inflammation in chronic GVHD. Bone Marrow Transplant 45: 957-958, 2010.

13. Abudou M, Wu T, Evans JR and Chen X: Immunosuppressants for the prophylaxis of corneal graft rejection after penetrating keratoplasty. Cochrane Database Syst Rev: CD007603, 2015.

14. Yamazoe K, Yamazoe K, Yamaguchi T, Omoto $M$ and Shimazaki J: Efficacy and safety of systemic tacrolimus in high-risk penetrating keratoplasty after graft failure with systemic cyclosporine. Cornea 33: 1157-1163, 2014.

15. Miedziak AI, Miller MR, Rapuano CJ,Laibson PR and Cohen EJ: Risk factors in microbial keratitis leading to penetrating keratoplasty. Ophthalmology 106: 1166-1171, 1999.

16. Skeens HM and Holland EJ: Large-diameter penetrating keratoplasty: Indications and outcomes. Cornea 29: 296-301, 2010.

17. Jia Y, Gao H, Li S and Shi W: Combined anterior chamber washout, amniotic membrane transplantation, and topical use of corticosteroids for severe peripheral ulcerative keratitis. Cornea 33: 559-564, 2014.

18. Cohen EJ, Kenyon KR and Dohlman CH: Iridoplasty for prevention of post-keratoplasty angle closure and glaucoma. Ophthalmic Surg 13: 994-996, 1982.

19. Mannis MJ and Holland EJ: Cornea-surgery of the cornea and conjunctiva. Fourth edition. Elsevier 1,2: pp1952, 2016.

20. Kharod-Dholakia B, Randleman JB, Bromley JG and Stulting RD: Prevention and treatment of corneal graft rejection: Current practice patterns of the Cornea Society (2011). Cornea 34: 609-614, 2015.

21. Jabs DA, Nussenblatt RB and Rosenbaum JT; Standardization of Uveitis Nomenclature (SUN) Working Group: Standardization of uveitis nomenclature for reporting clinical data. Results of the first international workshop. Am J Ophthalmol 140: 509-516, 2005.

22. Sloper CM, Powell RJ and Dua HS: Tacrolimus (FK506) in the management of high-risk corneal and limbal grafts. Ophthalmology 108: 1838-1844, 2001.

23. Rinne JR and Stulting RD: Current practices in the prevention and treatment of corneal graft rejection. Cornea 11: 326-328, 1992.

24. Williams KA, Roder D, Esterman A, Muehlberg SM and Coster DJ: Factors predictive of corneal graft survival. Report from the Australian corneal graft registry. Ophthalmology 99: 403-414, 1992
25. Panda A, Vanathi M, Kumar A, Dash Y and Priya S: Corneal graft rejection. Surv Ophthalmol 52: 375-396, 2007.

26. Snyder RW, Sherman MD and Allinson RW: Intracameral tissue plasminogen activator for treatment of excessive fibrin response after penetrating keratoplasty. Am J Ophthalmol 109: 483-484, 1990.

27. Yu Y, Zhong J, Peng L, Wang B, Li S, Huang H, Deng Y, Zhang H, Yang R, Wang $\mathrm{C}$ and Yuan J: Tacrolimus downregulates inflammation by regulating pro-/anti-inflammatory responses in LPS-induced keratitis. Mol Med Rep 16: 5855-5862, 217.

28. Sakuma S, Higashi Y, Sato N, Sasakawa T, Sengoku T, Ohkubo Y, Amaya T and Goto T: Tacrolimus suppressed the production of cytokines involved in atopic dermatitis by direct stimulation of human PBMC system. (Comparison with steroids). Int Immunopharmacol 1: 1219-1226, 2001.

29. Flower RJ: Eleventh Gaddum memorial lecture. Lipocortin and the mechanism of action of the glucocorticoids. Br J Pharmacol 94: 987-1015, 1988.

30. Ho S, Clipstone N, Timmermann L, Northrop J, Graef I, Fiorentino D, Nourse J and Crabtree GR: The mechanism of action of cyclosporin A and FK506. Clin Immunol Immunopathol 80: S40-S45, 1996.

31. Küchle M, Nguyen NX and Naumann GO: Aqueous flare following penetrating keratoplasty and in corneal graft rejection. Arch Ophthalmol 112: 354-358, 1994.

32. Xie L and Shi W: Comparison of immunological response after penetrating keratoplasty for three kinds of infectious corneal ulcers. Chin J Ophthalmol 18: 249-251, 2000 (In Chinese).

33. Dandona L, Naduvilath TJ, Janarthanan M, Ragu K and Rao GN: Survival analysis and visual outcome in a large series of corneal transplants in India. Br J Ophthalmol 81: 726-731, 1997.

34. Weisbrod DJ, Sit M, Naor J and Slomovic AR: Outcome of repeat penetrating keratoplasty and risk factors for graft failure. Cornea 22: 429-434, 2003.

35. Li C, Zhao GQ, Che CY, Lin J, Li N, Jia WY, Zhang QQ, Jiang N and Hu LT: Effect of corneal graft diameter on therapeutic penetrating keratoplasty for fungal keratitis. Int J Ophthalmol 5: 698-703, 2012.

36. Price MO, Thompson RW Jr and Price FW Jr: Risk factors for various causes of failure in initial corneal grafts. Arch Ophthalmol 121: 1087-1092, 2003

37. Sukhija $J$ and Jain AK: Outcome of therapeutic penetrating keratoplasty in infectious keratitis. Ophthalmic Surg Lasers Imaging 36: 303-309, 2005.

This work is licensed under a Creative Commons Attribution-NonCommercial-NoDerivatives 4.0 International (CC BY-NC-ND 4.0) License. 